\title{
Pancreatic Allografts: Identification of Useful Biomarkers of Rejection and Beyond
}

\author{
Charlotte Lawson
}

Published online: 8 January 2014

(c) Springer Science+Business Media New York 2014

Since organ transplantation became routine more than three decades ago, there has been a need to obtain useful information regarding graft health, with assessment of the presence of progressive immune-mediated graft rejection of particular importance for planning intervention. Although the recommended method is to obtain sequential biopsies from the graft itself, this procedure is invasive and risky, particularly if more than one sample is needed. Therefore, identification of less invasive markers, ideally measureable in the blood of transplant recipients, has been actively investigated. Because of the large number of losses of pancreatic grafts, it is particularly important to discover surrogate markers of immune rejection.

In this issue of Digestive Diseases and Sciences, Dong et al. [1] describe a porcine model of pancreaticoduodenal transplantation with or without immunosuppression that was used to evaluate the effectiveness of three potential peripheral blood biomarkers of acute rejection for up to seven days post-transplant. The authors selected intercellular adhesion molecule (ICAM)-1, Fas cell surface death receptor (Fas), and its ligand FasL, all reported previously to be involved in the acute rejection response after solid organ transplantation in humans and in animal models.

ICAM-1 is an adhesion molecule involved in firm endothelial adhesion and trans-endothelial migration of leukocytes, including $\mathrm{T}$ cells, monocytes, and neutrophils, at sites of immune response and immunological synapse formation (reviewed elsewhere [7]). ICAM-1 is

C. Lawson $(\bowtie)$

Department of Comparative Biomedical Sciences, Royal

Veterinary College, Royal College Street, London NW1 0TU, UK

e-mail: chlawson@rvc.ac.uk constitutively expressed on the surface of human endothelial cells and its expression is increased in the presence of pro-inflammatory cytokines including tumor necrosis factor (TNF)- $\alpha$, interferon (IFN)- $\gamma$ and interleukin (IL)- $1 \beta$. The most important ligand for ICAM-1 is the $\beta$ - 2 integrin lymphocyte function-associated (LFA)-1 ( $\alpha \mathrm{L} \beta 2)$; it is expressed on all types of leukocyte but requires a $\mathrm{Ca}^{2+}$. dependent conformational change before binding. Importantly, ICAM-1 expression on the surface of cardiac endothelium is increased as a consequence of rejection of human cardiac allografts [11] and has also been identified in pancreatic allografts [13]. The presence of the soluble form and antibodies to ICAM- 1 have also been detected in the serum of these patients [3,6]. Moreover, the functional relevance of increased ICAM-1 expression during rejection has been demonstrated using ICAM-1 knockout mice in models of heterotopic cardiac transplantation [5] whereas in a rat pancreas transplant model, treatment with antiICAM-1 antibodies attenuated acute allograft rejection [9].

Fas, also known as CD95 or Apo-1, is sometimes termed a "death receptor" because it can induce apoptosis in the cells on which it is expressed, after ligation by its cellsurface ligand FasL, highly regulated expression of which occurs on the surface of effector T cells. Expression of Fas and FasL have been identified in biopsies during acute rejection of cardiac allografts [8]. Furthermore, FasL induces tolerance in a number of transplant models including pancreatic islet models, after overexpression of the soluble protein [2, 4].

Dong et al. [1] found that all three molecules have potential as early indicators of acute rejection of pancreatic allografts; this must now be confirmed in biopsies and in the circulation of pancreatic transplant recipients. Their development of this porcine model suggests it could be a useful human correlate with potential for future discovery 
of increasingly sophisticated diagnostic biomarkers, but also as a model in which to trial novel anti-rejection therapeutics.

Since ICAM-1-LFA-1 interactions are involved in leukocyte firm adhesion to the endothelium and subsequent trans-endothelial migration and in immunological synapse formation, blocking the early post-transplant ICAM-1LFA-1 interactions between recipient leukocytes and graft vascular endothelial cells could potentially reduce the opportunities for reactive donor $\mathrm{T}$ cells to infiltrate the graft and become activated. Such an approach could therefore ultimately reduce acute rejection with the potential to induce tolerance of the transplanted graft. Thus, several years ago, there was a flurry of interest in the therapeutic use of antibodies to ICAM-1 to prevent acute and chronic allograft rejection. Results from initial animal studies were promising, although it was later reported that blocking antibodies to ICAM-1 themselves activated human neutrophils [12] and did not prevent acute rejection in a renal transplant trial [10].

Identification of increased expression of ICAM-1 preceding histopathologic signs of acute rejection in the transplanted pancreas, and, simultaneously, its increased expression in circulating leukocytes, immediately suggests a new possibility of preventing acute allograft rejection. Given recent advances in gene therapy, in particular development of small interfering RNA (siRNA) technology coupled with more efficient viral and non-viral approaches to gene transfer, it may be possible, in the not too distant future, to knock down ICAM-1 expression in donor endothelial cells and in recipient peripheral blood $\mathrm{T}$ lymphocytes before transplantation. Such an approach is attractive, because it may not only prevent acute rejection but could also affect chronic vascular rejection, thus improving long-term graft survival and, therefore, patient care.

\section{References}

1. Dong GH, Li XF, et al. Intercellular adhesion molecular-1, Fas, and Fas ligand as diagnostic biomarkers for acute allograft rejection of pancreaticoduodenal transplantation in pigs. Dig Dis Sci. (Epub ahead of print). doi:10.1007/s10620-013-2904-6.

2. Gainer AL, Suarez-Pinzon WL, et al. Improved survival of biolistically transfected mouse islet allografts expressing CTLA4-Ig or soluble Fas ligand. Transplantation. 1998;66:194-199.

3. Grant SC, Lamb WR, et al. Serum soluble adhesion molecules and cytokines in cardiac allograft rejection. The Manchester transplant cytokine group. Transpl Immunol. 1994;2:321-325.

4. Kawamoto K, Tanemura M, et al. Adenoviral-mediated overexpression of either membrane-bound human FasL or human decoy Fas can prolong pig islet xenograft survival in a rat transplant model. Transplant Proc. 2008;40:477-479.

5. Lacha J, Bushell A, et al. Intercellular cell adhesion molecule-1 and selectin ligands in acute cardiac allograft rejection: a study on gene-deficient mouse models. J Leukoc Biol. 2002;71:311-318.

6. Lawson C, Holder AL, et al. Anti-intercellular adhesion molecule-1 antibodies in sera of heart transplant recipients: a role in endothelial cell activation. Transplantation. 2005;80:264-271.

7. Lawson C, Wolf S. ICAM-1 signaling in endothelial cells. Pharmacol Rep. 2009;61:22-32.

8. Perez EC, Shulzhenko N, et al. Expression of Fas, FasL, and soluble Fas mRNA in endomyocardial biopsies of human cardiac allografts. Hum Immunol. 2006;67:22-26.

9. Preissler G, Eichhorn M, et al. Intercellular adhesion molecule-1 blockade attenuates inflammatory response and improves microvascular perfusion in rat pancreas grafts. Pancreas. 2012;41:1112-1118.

10. Salmela K, Wramner $\mathrm{L}$, et al. A randomized multicenter trial of the anti-ICAM-1 monoclonal antibody (enlimomab) for the prevention of acute rejection and delayed onset of graft function in cadaveric renal transplantation: a report of the European anti-ICAM-1 renal transplant study group. Transplantation. 1999;67:729-736.

11. Taylor PM, Rose ML, et al. Induction of vascular adhesion molecules during rejection of human cardiac allografts. Transplantation. 1992;54:451-457.

12. Vuorte J, Lindsberg PJ, et al. Anti-ICAM-1 monoclonal antibody R6.5 (Enlimomab) promotes activation of neutrophils in whole blood. J Immunol. 1999;162:2353-2357.

13. Wiessner R, Eisold S, et al. Up-regulation of ICAM-1 during cold ischemia triggers early neutrophil infiltration in human pancreas allograft reperfusion. Transplant Proc. 2009;41:3622-3627. 